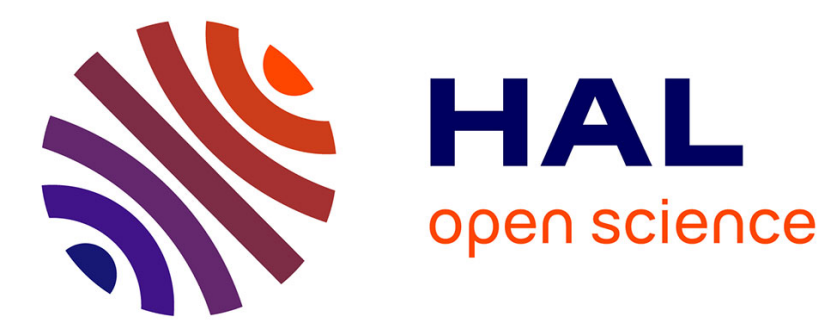

\title{
Risk Assessment for Collision Avoidance Systems
} Adam Houénou, Philippe Bonnifait, Véronique Cherfaoui

\section{To cite this version:}

Adam Houénou, Philippe Bonnifait, Véronique Cherfaoui. Risk Assessment for Collision Avoidance Systems. IEEE 17th International Conference on Intelligent Transportation Systems (ITSC 2014), Oct 2014, Qingdao, China. pp.386-391. hal-01079722

\section{HAL Id: hal-01079722 \\ https://hal.science/hal-01079722}

Submitted on 3 Nov 2014

HAL is a multi-disciplinary open access archive for the deposit and dissemination of scientific research documents, whether they are published or not. The documents may come from teaching and research institutions in France or abroad, or from public or private research centers.
L'archive ouverte pluridisciplinaire HAL, est destinée au dépôt et à la diffusion de documents scientifiques de niveau recherche, publiés ou non, émanant des établissements d'enseignement et de recherche français ou étrangers, des laboratoires publics ou privés. 


\title{
Risk Assessment for Collision Avoidance Systems
}

\author{
Adam Houénou*, Philippe Bonnifait ${ }^{+}$and Véronique Cherfaoui ${ }^{+}$
}

\begin{abstract}
Collision Avoidance Systems need to perform scene analysis and risk assessment in order to react conveniently. Based on the information provided by the perception system, scene analysis has to predict the evolution of the current driving situation for the near future. Thanks to the predicted trajectories of the relevant traffic participants, the risk of collision on the ego vehicle can be calculated. In many cases, a predicted trajectory is not defined with explicit equations but is given as a set of sampled poses, each one corresponding to a different future time instant. A predicted trajectory being always uncertain, confidence has to be estimated on the so predicted poses. We present a method that propagates the known error covariance matrix of the current pose of the ego vehicle by considering local approximations of the predicted trajectory. This allows to estimate the risk of collision of the ego vehicle with a considered target object. The proposed approach uses a Monte Carlo simulation to approximate the probability that the ego vehicle and the object come into collision at a given future time instant. Each sample time of the whole prediction horizon is considered as a potential collision time so that a curve describing the variation of the risk of collision is obtained. This allows the system to have a better comprehension of the scene and to react proportionally to the threat. The overall approach has been tested with simulated data and the consistency of results is shown.
\end{abstract}

\section{INTRODUCTION}

Driver Assistant Systems in general and more specifically Collision Avoidance Systems (CASs) are very active fields of research and development. Recent years have seen many of those systems on the market of series vehicles. A CAS usually performs in low speed scenarios but enhancements are under research in order to cover more scenarios. A CAS needs to predict any eventual collision as soon as possible and must warn the driver or take autonomous actions in order to avoid it or mitigate its consequences. For this purpose, it has to rely first on an accurate perception system that uses sensors, communication devices and data fusion algorithms to detect and track obstacles in the surrounding of the ego vehicle. Then, it is crucial to perform a correct scene analysis which consists in predicting the evolution of the scene. Then, it is possible to assess the risk of collision of the ego vehicle. Depending on the risk assessment result, various criteria can be used to decide the relevant actions that have to be finally executed.

This paper first focuses on the scene analysis part. Based on the estimated current and past states of the scene, the goal here is to understand the current driving situation and

\footnotetext{
*Sherpa Engineering (France), a.houenou@sherpa-eng.com ${ }^{+}$Heudiasyc UMR CNRS 7253, University of Technology of Compiègne (France)
}

to use this result to predict the most probable evolution of the scene. In a previous work [1], we described a trajectory prediction method based on the combination of two predictions made with different approaches. The first prediction uses a deterministic approach. Its assumes a kinematic motion model to predict the trajectory of a vehicle. The second prediction is based on a maneuver recognition algorithm, a trajectory generation process and a cost function used to select the best trajectory. A combination rule allows the final prediction to take advantage of the good accuracy of the motion model approach for short term prediction and of the better accuracy of the maneuver recognition method for longer term prediction. Section II gives a reminder of this prediction method in order to make this paper more intelligible.

Based on this information, the CAS has to evaluate the threat on the ego vehicle in the near future in order to be able to avoid an eventual collision with any potential obstacle. This is the task of the risk assessment module that takes as inputs the predicted trajectory of the ego vehicle and the predicted trajectories of the detected objects. Since these predictions are not deterministic, it is necessary to estimate their uncertainty. A first contribution of this paper is the presentation of a method that propagates the estimated uncertainty of the current pose of a given vehicle along its predicted trajectory that is time sampled and that has no closed-form. This is done by local linearizations of the predicted trajectory and a sequential estimation of the covariance matrices of its consecutive future sample poses. This is described in section III.

Many risk assessment approaches exist in the literature [3], [6], [5], [7], [8] but the notion of risk of collision is usually related to the time-to-collision (TTC) or other derived time metrics. The smaller the TTC, the higher the risk. The predicted trajectories being uncertain, the TTC is also uncertain. The proposed approach consists in sampling the prediction time horizon and considering each sample time as a potential TTC. Then, for each sample time and each target object, the probability that the ego vehicle is in collision with the object is calculated. This gives a pseudo-continuous evolution of the risk of collision with each obstacle on the whole prediction horizon which allows the system to define various levels of criticality based on the pairs TTC/probability of collision. The risk assessment method is presented in section IV and its principle is compared to known approaches in the literature. Section V shows the evaluation of the overall risk assessment method in simulated scenarios and the results are discussed. Finally, section VI concludes the paper. 


\section{TRAJECTORY PREDICTION}

From the driver's control commands to the actual trajectory of the vehicle, there are complex interactions including the vehicle's dynamic and the road's structure. Predicting the trajectory of a vehicle (either the ego-vehicle or any detected vehicle) is not a deterministic task because the driver's control commands are quite unpredictable and many parameters are difficult to measure in normal conditions e.g. tire friction on the road. In some works [11], [8], [2], the prediction is made by assuming a kinematic model of the vehicle's motion. Unfortunately, such motion models as constant velocity or constant yaw rate movement are accurate only for a short term, except if the driver's command actually remains unchanged for a long time which is unlikely. During a lane change maneuver for instance, the movement changes a lot and the prediction can quickly be very wrong. In other papers, a Maneuver Recognition Module (MRM) is used to compute a predicted trajectory that takes into account the possible changes in the vehicle's behavior with respect to the recognized maneuver. In most cases [6], [4], [12], [10], the MRM is based on a set of learning trajectories. The current recorded sequence of the vehicle's trajectory is compared to that database according to various possible techniques and the most likely future trajectory is obtained from the closest trajectories in the database.

In [1], we proposed a new method that mixes both approaches in order to take advantage on the high accuracy of the motion model method for short-term prediction and to respect, for long-term prediction, the possibly changing dynamic of the vehicle during specific maneuvers. Our MRM is not based on a data set; it uses the last measurements of kinematic parameters and the knowledge of the road geometry to decide whether the vehicle will stand in its current lane or if it is doing a lane change maneuver. This is done by comparing local parabolic models of the lane's center-line and of the trajectory.

Knowing the vehicle's current state and its future lane, several possible maneuver's end points are generated by sampling in a limited area. Then, a set of trajectories are generated by joining the current vehicle's position to each end point. The trajectories are calculated so that their longitudinal and their lateral components are quintic time polynomials in the Frenet frame along the center-line of the vehicle's current lane. The MRM-based predicted trajectory is selected as the best one, with respect to a cost function that penalizes timetaking trajectories and trajectories with high pick of lateral acceleration, synonym of low comfort in the cockpit. Fig.1 shows an example of generated trajectories and the selected one for a lane change maneuver. Even though the trajectories are defined with explicit polynomials, the conversion from the Frenet frame to the working Cartesian frame does not have an analytical solution if the shape of the road is not a straight line or a perfect circle (see appendix of [1] for the conversion method). So, the trajectory is sampled and the conversion is made point by point. The MRM-based trajectory prediction (denoted $T_{\text {man }}$ in the following) is thus

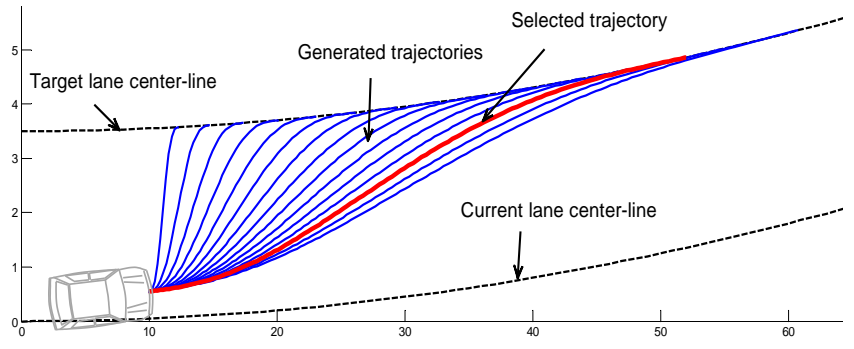

Figure 1. MRM-based trajectory prediction

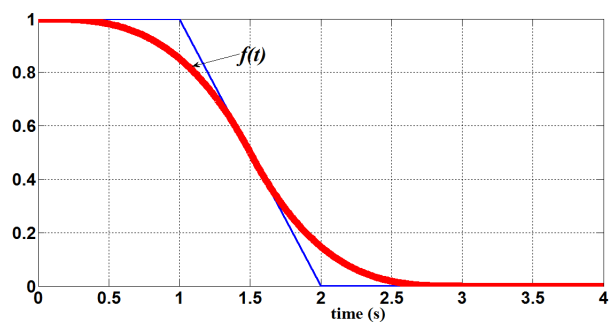

Figure 2. Cubic spline used to combine $T_{m d l}$ and $T_{m a n}$. At short term, $T_{f i n}$ is closer to $T_{m d l}$ and progressively gets close to $T_{\text {man }}$ as the prediction time increases.

a sequence of predicted poses with their associated time instant.

The assumed motion model is the Constant Yaw Rate and Acceleration (CYRA) movement. It is defined with a sixcomponent state vector $X=[x, y, \theta, v, a, \omega]^{T}$ where $(x, y)$ is the position in the considered Cartesian frame, $\theta$ the yaw angle, $v$ and $a$ the longitudinal velocity and acceleration and $\omega$ the yaw rate. Defining $X_{0}$ as the current estimated state, Eq. 1 gives the closed-form of the predicted trajectory (denoted $T_{m d l}$ in the following). Index 0 expresses the current estimated values.

$$
T_{m d l}:\left\{\begin{array}{l}
x(t)=\frac{a_{0}}{\omega_{0}^{2}} \cos (\theta(t))+\frac{v(t)}{\omega_{0}} \sin (\theta(t))+c x \\
y(t)=\frac{a_{0}}{\omega_{0}^{2}} \sin (\theta(t))-\frac{v(t)}{\omega_{0}} \cos (\theta(t))+c y \\
\theta(t)=\omega_{0} \cdot t+\theta_{0} \\
v(t)=a_{0} \cdot t+v_{0} \\
a(t)=a_{0} \\
\omega(t)=\omega_{0}
\end{array}\right.
$$

where $c x$ and $c y$ are constant values obtained from the current state's values.

$$
\left\{\begin{array}{l}
c x=x_{0}-\frac{v_{0}}{\omega_{0}} \sin \left(\theta_{0}\right)-\frac{a_{0}}{\omega_{0}^{2}} \cos \left(\theta_{0}\right) \\
c y=y_{0}+\frac{v_{0}}{\omega_{0}} \cos \left(\theta_{0}\right)-\frac{a_{0}}{\omega_{0}^{2}} \sin \left(\theta_{0}\right)
\end{array}\right.
$$

The final predicted trajectory, denoted $T_{f i n}$ is a linear combination of $T_{m a n}$ and $T_{m d l}$ :

$$
T_{f i n}(t)=f(t) \cdot T_{m d l}(t)+(1-f(t)) \cdot T_{\text {man }}(t)
$$

where $f(t)$ is a predefined function as the one depicted in Fig.2. Fig.3 shows an example of a trajectory prediction. 


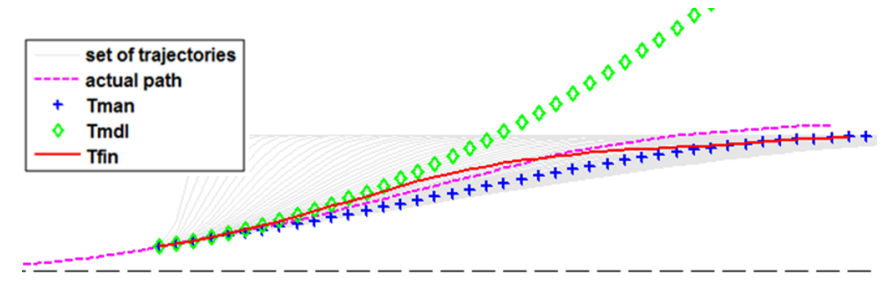

Figure 3. Example of a trajectory prediction at a given time during a lane change. $T_{m d l}$ gets wrong as time increases. $T_{f i n}$ is closer to the actual trajectory than each of $T_{m a n}$ and $T_{m d l}$.

One can notice that since we do not have an explicit expression of $T_{\text {man }}, T_{\text {fin }}$ is also a set of predicted sampled poses. The problem is now to estimate the covariance matrix of the prediction error of each future pose.

\section{PRopagation OF UNCERTAINTY}

Let's define $\xi=[x, y, \theta]^{T}$ as the pose of a considered vehicle at a given time. $(x, y)$ is the position in the working Cartesian frame and $\theta$ is the yaw angle. The estimated current pose $\xi_{0}$ and its error covariance matrix $\Sigma_{0}$ are known from the tracking system. Let $\xi^{N}=\left\{\xi_{1}, \xi_{2}, \cdots, \xi_{k}, \cdots \xi_{N}\right\}$ be the set of predicted poses with a sampling period denoted $T_{e}$. If the predicted trajectory was known in a closed-form as $\xi_{k+1}=$ $g\left(\xi_{k}\right)+\alpha$, with $g(\xi)$ a known function and $\alpha$ the model noise, the covariance matrix of $\xi_{k+1}$ could be calculated with a first order expansion:

$$
\Sigma_{k+1}=J \cdot \Sigma_{k} \cdot J^{T}+Q
$$

where $\Sigma_{k}$ and $Q$ are respectively the covariance matrices of the error on $\xi_{k}$ and of $\alpha . J=\frac{\partial g}{\partial \xi}\left(\xi_{k}\right)$ is the Jacobian of function $g$ in point $\xi_{k}$. In our case, such a $g$ function is not known. So, we first consider a new state vector $\mathbb{X}$ defined as

$$
\mathbb{X}=\left[\begin{array}{c}
\xi \\
\dot{\xi}
\end{array}\right]=\left[\begin{array}{c}
x \\
y \\
\theta \\
v_{x} \\
v_{y} \\
\omega
\end{array}\right]
$$

$v_{x}$ and $v_{y}$ are respectively the velocities along $x$-axis and $y$ axis, $\omega$ is the yaw rate. The error covariance matrix of $\mathbb{X}$ is denoted $\mathbb{P}$. The initial (current) values $\mathbb{X}_{0}$ and $\mathbb{P}_{0}$ are known from the tracking system. Then, we assume a constant yaw rate and velocity movement between two consecutive samples $\mathbb{X}_{k}$ and $\mathbb{X}_{k+1}$, so that $v_{x, k}$, for instance, is given by:

$$
v_{x, k}=\frac{x_{k+1}-x_{k}}{T_{e}}
$$

If one integrates along the trajectory with $x_{k+1}=x_{k}+$ $T_{e} \cdot v_{x, k}$ and develops this expression, one can check that we have $x_{k+1}=x_{k+1}$. Therefore, starting from $\mathbb{X}_{0}$, and having the same reasoning for all the state parameters, we get exactly the same points of the predicted trajectory.

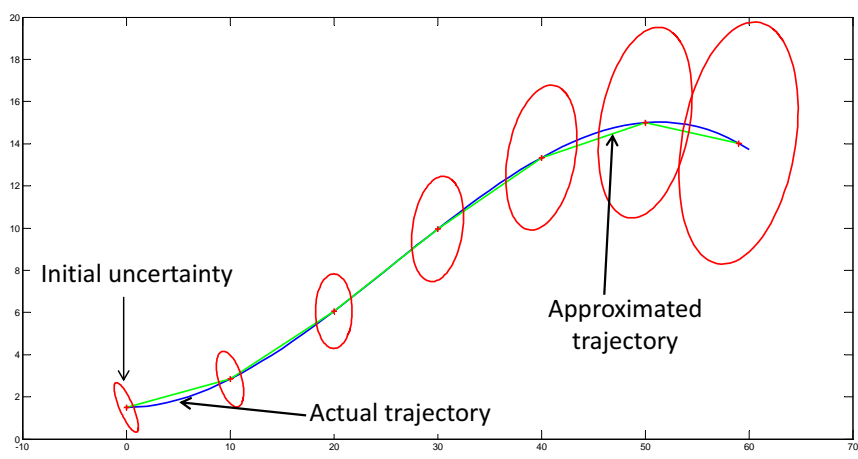

Figure 4. Illustration of the propagation of an initial uncertainty along a predicted trajectory.

The linear state evolution is:

$$
\mathbb{X}_{k+1}=A \cdot \mathbb{X}_{k}+\alpha
$$

where

$$
A=\left[\begin{array}{cccccc}
1 & 0 & 0 & T_{e} & 0 & 0 \\
0 & 1 & 0 & 0 & T_{e} & 0 \\
0 & 0 & 1 & 0 & 0 & T_{e} \\
0 & 0 & 0 & 1 & 0 & 0 \\
0 & 0 & 0 & 0 & 1 & 0 \\
0 & 0 & 0 & 0 & 0 & 1
\end{array}\right]
$$

and the zero-mean white model noise $\alpha$ is added to handle the fact that this model is an approximation of the real movement (Fig.4). In practice, the covariance matrix $Q$ of $\alpha$ is chosen constant with its first three diagonal elements null because the noise does not affect the next pose (the first three state elements) which is already known from the trajectory prediction. The error covariance matrix of $\mathbb{X}_{k+1}$ is given by:

$$
\mathbb{P}_{k+1}=A \cdot \mathbb{P}_{k} \cdot A^{T}+Q
$$

The covariance matrix $\Sigma_{k+1}$ of $\xi_{k+1}$ is finally extracted as the upper left block of $\mathbb{P}_{k+1}$.

Fig.4 shows a simplified example (with only position and velocity) of the propagation of the initial uncertainty along a predicted trajectory. In this example:

$$
\mathbb{P}_{0}=\left[\begin{array}{cccc}
1 & 0.81 & 0 & 0 \\
0.81 & 1 & 0 & 0 \\
0 & 0 & 1 & 0.64 \\
0 & 0.64 & 0 & 1
\end{array}\right], \quad Q=\left[\begin{array}{llll}
0 & 0 & 0 & 0 \\
0 & 0 & 0 & 0 \\
0 & 0 & 1 & 0 \\
0 & 0 & 0 & 1
\end{array}\right]
$$

The model noise here, is chosen pretty large in order to easily check the increase of the uncertainty, depicted by ellipses in the figure.

\section{ESTIMATION OF THE COLLISION RISK}

The risk of collision is computed separately for each obstacle in a limited area around the ego vehicle. In [6], the predicted trajectory of each considered object (ego vehicle or target object) is used as mean value of a Gaussian Process [9] 
and several random trajectory samples are generated for that object. Then, the fraction of the ego vehicle's trajectories that collide with one or more trajectories of a considered object gives the risk of collision with that object. This approach gives an idea of the collision risk on the whole predicted trajectory but does not provide an estimation of theTTC nor the point of the collision which are yet prime indicators, in the sense of collision avoidance.

In [3], the predicted trajectories are used to define a time function representing the distance between the ego vehicle and the considered obstacle. The smallest positive zero (if any) of this function stands for the TTC. Then, the predicted poses of both objects at that time and their variances are used to generate several Gaussian random poses. Finally, the fraction of poses that depict a collision gives the probability collision at that time. Here, the variances of the future poses are considered equal to their initial values (current time). This approach uses a deterministic method to determine the TTC, which might be a fail since the predicted trajectories are not certain. The actual TTC may potentially be different. Moreover, the variances actually increase in time.

In [5], the authors proposed a method to compute the probability of collision of two objects at a given moment, knowing an estimate of their respective poses at that time and the corresponding error covariance matrices. The poses are considered as Gaussian variables and the probability that the objects (named $n$ and $m$ ) share the same space is:

$$
P_{\text {coll }}=\int_{D} p_{n}(\xi) p_{m}(\xi) d x_{n} d y_{n} d \theta_{n} d x_{m} d y_{m} d \theta_{m}
$$

where the probability density functions $p_{n}$ and $p_{m}$ are Gaussian:

$$
p_{i}(\xi)=\frac{1}{(\sqrt{2 \pi})^{3} \sqrt{\left|\Sigma_{i}\right|}} e^{-\frac{1}{2}\left(\left(\xi-\xi_{i}\right)^{T} \Sigma_{i}^{-1}\left(\xi-\xi_{i}\right)\right)} \mid i=\{n, m\}
$$

and

$D=\left\{\left(x_{n}, y_{n}, x_{m}, y_{m}\right) \in \mathbb{R}^{4},\left(\theta_{n}, \theta_{m}\right) \in\left[0,2 \pi\left[^{2} \mid \mathcal{S}_{n} \cap \mathcal{S}_{m} \neq \emptyset\right\}\right.\right.$

$\mathcal{S}_{n}$ and $\mathcal{S}_{m}$ being the areas respectively occupied by objects $n$ and $m$. If $\mathcal{S}_{n}=\mathcal{S}_{m}=\emptyset$, Eq. 7 has an analytical solution, otherwise it does not exist. Since we consider objects with non null surface, the probability is computed numerically via a Monte-Carlo (MC) simulation, consisting in generating random poses for each object and computing the ratio of pairs of poses corresponding to a collision.

In a similar way to the previously cited paper, our approach is also based on a Monte Carlo simulation but it overcomes the weaknesses mentioned before. Its main steps are the following:

1) For each considered object (including ego vehicle), propagate the initial uncertainty along its predicted trajectory (see section III)

2) For each future time sample, compute the probability of collision of the ego vehicle with each target object.
The probability is approximated by generating random poses for the ego vehicle and for the object, based on their predicted poses and associated covariance at this time. Then, the ratio of cases corresponding to a collision is the probability of collision.

3) For each target object, output the set of probability values (corresponding to the different future time samples).

Instead of estimating the actual TTC for each target object, each future time sample is considered as a potential TTC and the probability of collision is computed. This approach allows to have a larger view of the evolution of the scene and to set up different strategies, according to the probability of collision for specific values of TTC.

\section{Simulation AND RESUlts}

The risk assessment approach has been tested in simulation. Two different use cases were evaluated, with the goal of checking the evolution of the probability of collision of the ego vehicle with each encountered object. Both simulation last $12 \mathrm{~s}$. There are 3 vehicles, the ego vehicle named $V_{0}$ and two target vehicles named $V_{1}$ and $V_{2}$. The curves representing the evolution of the risk of collision for the next $4 \mathrm{~s}$ are plotted all along each simulation, as shown in Fig.5 and Fig.6. The upper sub-graphs shows a bird view of the scenario at the mentioned time instant. We skip the letter " $V$ " in the name of the vehicles for a better readability of the figures. So, "0" stands for $V_{0}$, "1" for $V_{1}$ and so on. The middle and the bottom sub-graphs depict the risk of collision of $V_{0}$ respectively against $V_{1}$ and $V_{2}$. Both scenarios happen on a straight road with 2 lanes, each $3.5 \mathrm{~m}$ wide. The simulator provides position, velocity and orientation data for each vehicle, at a rate of $10 \mathrm{~Hz}$. At each sample time, a $4 \mathrm{~s}$ trajectory prediction is made for each object. Then, the predicted trajectories are sampled, also on a $10 \mathrm{~Hz}$ rate and the probability of collision of $V_{0}$ against each vehicle is computed for each corresponding pair of future sample poses. For the MC algorithm, only 100 drawings are made per predicted pose. This value may seem low but gives realistic results as discussed in the following subsections. Moreover, it is a good compromise to afford a real-time execution. One may try a higher number of drawings to have a better approximation of the integral in Eq. 7, if the required computational resources are available.

\section{A. Scenario 1: Collision during an overtaking}

In the first scenario, the velocities of $V_{0}, V_{1}$ and $V_{2}$ are respectively $80 \mathrm{~km} / \mathrm{h}, 50 \mathrm{~km} / \mathrm{h}$ and $40 \mathrm{~km} / \mathrm{h}$. At the beginning, all the vehicles are in the bottom lane. $V_{0}$ follows $V_{1}$ which follows $V_{2}$. Then, $V_{0}$ starts an overtaking on $V_{1}$ by making a lane change to the upper lane. While it gets close to $V_{1}$, the latter suddenly starts a lane change in order to overtake $V_{2}$. A collision happens in the upper lane, between $V_{0}$ and $V_{1}$ at time $7.4 \mathrm{~s}$. Fig.5 shows the risk of collision at different moments of the simulation. 

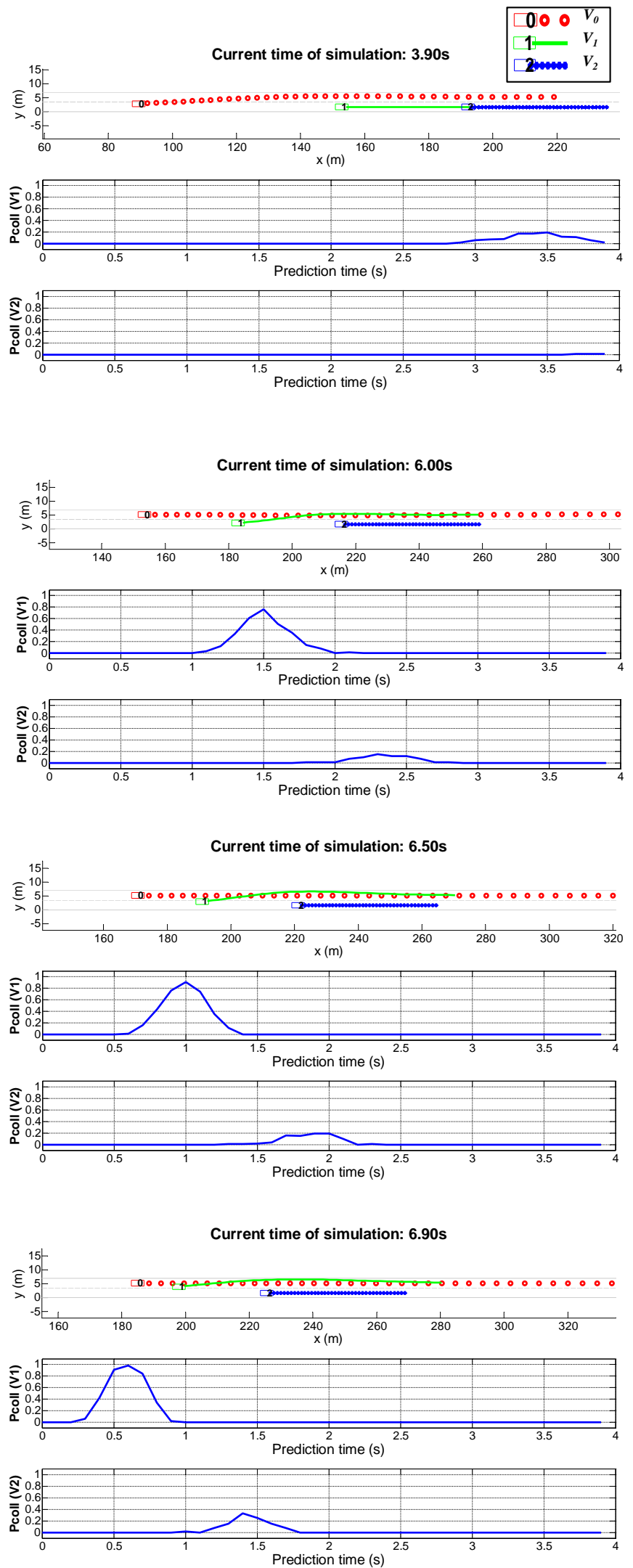

Figure 5. Probability of collision during simulation $\mathrm{N}^{\circ} 1$
As it can be noticed from Fig.5, the pick of probability of collision for $V_{1}$ is always around time 7.4s (when reading the figure, please add the prediction time to the current simulation time) and the value of the pick increases as the car gets closer to the moment of the collision. This is the desired behavior because it allows a CAS to react proportionally to the time before the collision. This also means that the trajectory prediction method and the collision risk estimation are consistent.

By checking the values of the curve for $V_{1}$, we have about $50 \%$ and $65 \%$ respectively $2 \mathrm{~s}$ and $1.5 \mathrm{~s}$ before the collision, which are medium levels of alert and leaves enough time to make a maneuver in order to avoid the collision. 1s before the collision, the probability reaches $80 \%$ and reaches $100 \%$ around $0.8 \mathrm{~s}$ meaning that the collision is very soon and that there are very few chances to avoid it if no action is taken immediately.

Regarding $V_{2}$, the risk of collision is not null despite the fact that it is not in the same lane as $V_{0}$. Indeed, when they are predicted to be almost side by side or at least pretty close to each other, the variances of the predicted poses leave a few chances to have a collision. Nevertheless, the maximal pick is $40 \%$ and the mean value along the whole simulation is less than $20 \%$ which means that the risk of collision between $V_{0}$ and $V_{2}$ remains, as expected, low.

\section{B. Scenario 2: Front to front collision narrowly avoided}

In the second scenario, $V_{0}$ follows $V_{1}$ in the bottom lane while $V_{2}$ is driving in the upper lane but in the opposite direction. They respectively drive at $50 \mathrm{~km} / \mathrm{h}, 40 \mathrm{~km} / \mathrm{h}$ and $40 \mathrm{~km} / \mathrm{h}$. Then, $V_{0}$ starts a lane change in order to overtake $V_{1}$.

A collision has almost happened between $V_{0}$ and $V_{2}$ shortly later but has narrowly been avoided because $V_{0}$ came back to the bottom lane just in time. The goal here, is to check the behavior of the system when the driving behavior changes. Fig.6 shows the risk of collision at different moments of the simulation.

In this scenario, there was no collision and, as expected, the risk of collision with $V_{2}$ never reached $100 \%$. It even remains quite low despite the fact that both vehicle happened to be driving in opposite directions in the same lane. This is so because at the beginning, even though they were in the same lane, they are pretty far from each other (more than $150 \mathrm{~m}$ in the first sub-figure of Figure 6).

Later, the lane change made by $V_{0}$ has been detected early making their predicted trajectories not meet. However, as the vehicles get closer, the risk of collision increases. The pick values reached in the two bottom sub-figures of Figure 6 show that they were close to a collision. Please notice that the trajectory prediction is crucial to obtain a realistic result. For $V_{1}$ the analysis is similar to the one for $V_{2}$ in the first scenario. 

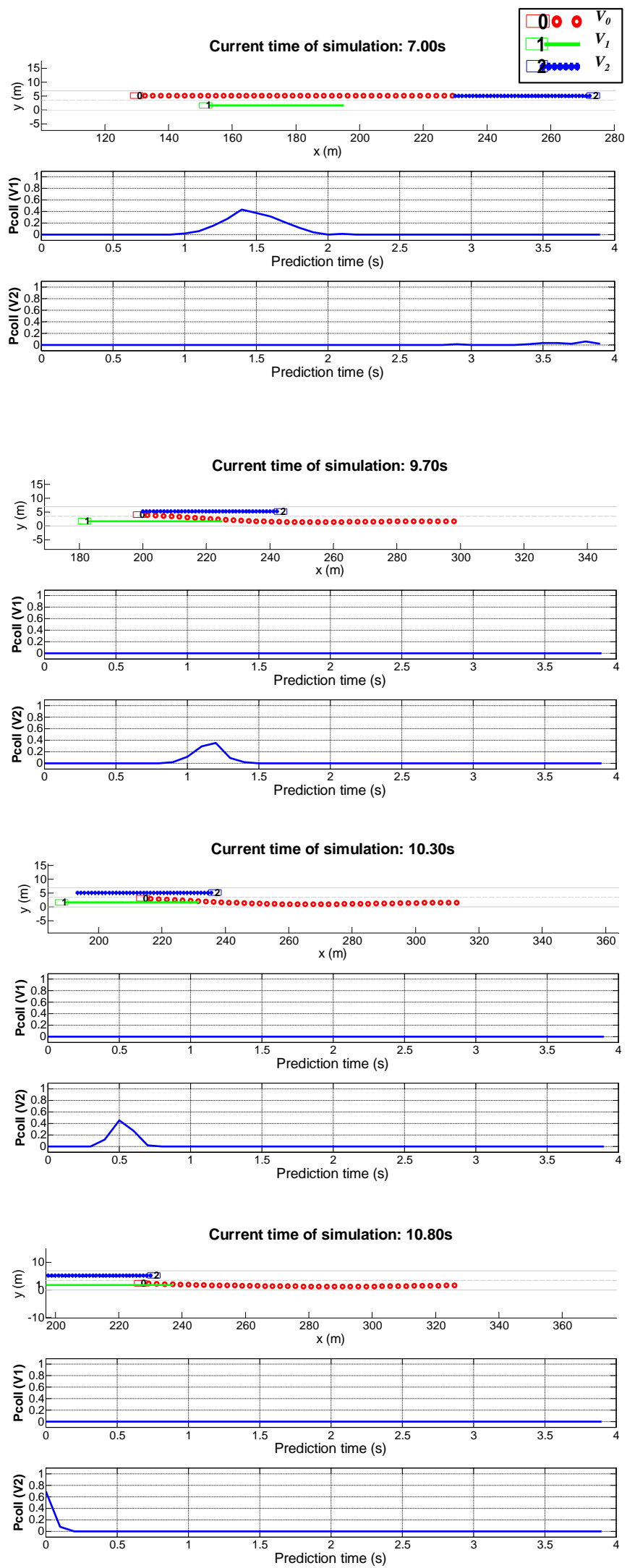

Figure 6. Probability of collision during simulation $\mathrm{N}^{\circ} 2$

\section{CONCLUSION}

This paper presented an approach to assess the risk of collision of the ego vehicle in a dynamic scene. This, first includes object trajectory predictions. So, we reminded a trajectory prediction method which was used later in the evaluation of the approach. In order to calculate the risk of a predicted collision, it is crucial to know the uncertainty of the predicted poses. A method was presented to propagate the estimated uncertainty of the current pose of a vehicle along its predicted trajectory. Then, a method to approximate the risk of collision between two objects at a given time was shown. It is based on a Monte Carlo strategy. The global idea of the risk assessment is to have a pseudo-continuous evolution of the probability of collision on the whole prediction horizon so that it possible for a Collision Avoidance System to set up different action strategies depending on the value of the probability at specific future time instants. The overall approach was tested in two different scenarios and the results show that the output is very realistic which should allow to reduce false alarms in a CAS when the driving behavior changes before a coming collision.

\section{REFERENCES}

[1] A.Houenou, P. Bonnifait, V. Cherfaoui, and W. Yao. Vehicle trajectory prediction based on motion model and maneuver recognition. IEEE/RJS International Conference on Intelligent Robots and Systems, Tokyo, Japan, pages 4363-4369, Nov 2013.

[2] D. Althoff, M. Werling, N. Kaempchen, D. Wollherr, and M. Buss. Lane-based safety assessment of road scenes using inevitable collision states. IEEE Intelligent Vehicles Symposium, Alcala de Henares, Spain, pages 31-36, june 2012.

[3] A. Berthelot, A. Tamke, T. Dang, and G. Breuel. Handling uncertainties in criticality assessment. IEEE Intelligent Vehicles Symposiun (IV), Baden-Baden, Germany, 2011.

[4] T. Hülnhagen, I. Dengler, A. Tamke, T. Dang, and G. Breuel. Maneuver recognition using probabilistic finite-state machines and fuzzy logic. IEEE Intelligent Vehicles Symposium, San Diego, CA, USA, June 2010.

[5] A. Lambert, D. Gruyer, and G. Saint Pierre. A fast monte carlo algorithm for collision probability estimation. International Conference on Control, Automation, Robotics and Vision, Hanoi, Vietnam, Dec 2008.

[6] C. Laugier, Igor E. Paromtchik, M. Perrollaz, J.-D. Yoder, C. Tay, M. Yong, A. Nègre, and K. Mekhnacha. Probabilistic analysis of dynamic scenes and collision risks assessment to improve driving safety. IEEE Intelligent Transportation Systems Magazine, 3:4 - 19, 2011.

[7] S. Lefèvre, C. Laugier, and J. Ibañez-Guzmán. Risk assessment at road intersections: Comparing intention and expectation. IEEE Intelligent Vehicles Symposium, Alcala de Henares, Spain, pages 165-171, June 2012.

[8] A. Polychronopoulos, M. Tsogas, U. Scheunert, L. Andreone, and F. Tango. Dynaminc situation and threat assessment for collision warning systems: the euclide approach. IEEE Intelligent Vehicles Symposium Parma, Italy, June 2004.

[9] C. Rasmussen and C. Williams. Gaussian processes for machine learning. Massachusetts Institute of Technology, The MIT Press, 2006. ISBN 026218253X.

[10] C. Sung, D. Feldman, and D. Rus. Trajectory clustering for motion prediction. IEEE/RJS International Conference on Intelligent Robots and Systems, Vilamoura, Portugal, Oct 2012.

[11] A. Tamke, T. Dang, and G. Breuel. A flexible method for criticality assessment in driver assistance systems. IEEE Intelligent Vehicles Symposium Baden-Baden, Germany, June 2011.

[12] J. Wiest, M. Höffken, U. Kreßel, and K. Dietmayer. Probabilistic trajectory prediction with gaussian mixture models. IEEE Intelligent Vehicles Symposium, Alcala de Henares, Spain, June 2012. 